\title{
Participación de los gobiernos regionales en la definición de políticas públicas en Chile: el caso del gobierno regional de Los Lagos y la política nacional de acuicultura*
}

\author{
Alejandro Santibáñez Handschuh** \\ Mauricio Barra Ranni***
}

\begin{abstract}
Sumario: 1. Elementos introductorios; 2. Las políticas públicas y los actores en democracia; 3. Políticas públicas y descentralización; 4. La descentralización en el nivel regional: elementos para entender la falta de participación de los Gores en la construcción y generación de políticas públicas; 5 . Descripción del caso: la Política Nacional de Acuicultura y la participación del Gore de Los Lagos y SalmonChile en su definición; 6. Aspectos metodológicos; 7. Presentación de los resultados; 8. A modo de conclusiones.
\end{abstract}

Summary: 1 . Introductory elements; 2 . Public policy and the actors in democracy; 3. Public policy and decentralization; 4. Decentralization at the regional level: elements to understand the lack of participation of the regional governments in the construction and generation of public policy; 5. Description of the case: national policy of hydroponics and the participation of the Los Lagos regional government and SalmonChile in its definition; 6. Methodology; 7. Presentation of the results; 8. Conclusions.

\footnotetext{
* Artículo recibido en septiembre 2007 e acepto en enero 2008.

** Profesor, investigador y consultor en las áreas de gobierno y administración regional, gestión y políticas públicas de la Escuela de Ciencias Políticas y Administrativas de la Universidad de Los Lagos en Puerto Montt - Chile, autor de numerosas publicaciones en su especialidad, investigador principal en proyectos Fondecyt y Fondef de la Comisión Nacional de Investigación Científica y Tecnológica del Estado de Chile (Conicyt), fundador y director de la Revista Chilena de Gobierno y Empresa, se desempeña en la actualidad como vicerrector de Gestión Corporativa de la Universidad de Los Lagos. Dirección: Chinquihue Km 6, Puerto Montt, Chile. Correo electrónico: asantiba@ulagos.cl.

**** Administrador público y licenciado en administración pública de la Universidad de Los Lagos, investigador del Observatorio de Políticas Públicas y profesional del Centro de Recursos del Aprendizaje del Campus Puerto Montt de la misma universidad. Dirección: Chinquihue Km 6, Puerto Montt, Chile. Correo electrónico: mauriciobarra@ulagos.cl.
} 
Palabras clave: políticas públicas; descentralización; gobierno regional.

KEY WORDs: public policies; decentralization; regional government.

El artículo aporta evidencia respecto de la participación de los gobiernos regionales en la definición de políticas públicas en Chile a través del caso del gobierno regional de Los Lagos. Se evalúa la participación de los principales actores regionales en la definición de políticas públicas como indicador del grado de centralización en el diseño político institucional en Chile. La metodología utilizada fue el análisis documental relacionado con el caso presentado, el conocimiento del estado del arte respecto del análisis de políticas públicas como disciplina y la descentralización, posteriormente se contrastaron las hipótesis que guiaron el estudio a través del análisis de discurso aplicado a las entrevistas de los actores que estuvieron involucrados en la definición de la Política Nacional de Acuicultura.

Participation of regional governments in the definition of public policy in Chile: the case of Los Lagos regional government and the National Policy of Hydroponics

The article treats the participation of regional governments in the definition of public policy in Chile through the case of Los Lagos Regional Government. It evaluates the participation of the principal regional actors in the definition of public policy as indicators of the degree of centralization in the political institutional design of Chile. The methodology used was the documentary analysis related to the illustrated case, the knowledge of the state refering to the analysis of public policy as a discipline, and the decentralization. Later it was shown the hypotheses that guided the study through the analysis of discourse applied to the interviews of the actors who were involved in the definition of National Policy of Hydroponics.

\section{Elementos introductorios}

Una vez restituida la democracia en Chile, los gobiernos de la coalición gobernante han definido como uno de sus grandes desafíos la profundización y el fortalecimiento de la regionalización. En función de lo anterior, se han implementando varias reformas orientadas a fortalecer el proceso de descentralización, que ha adoptado como principios rectores la equidad, la participación y la autonomía en la acción de las entidades subnacionales. No obstante, se observa que descentralización y regionalización aun son conceptos que no están en sintonía con el relato de una democracia participativa. Según el informe final sobre "Medición y evaluación del proceso de descentralización en Chile" (Gobierno de Chile, 2004), se establece que la relación Estado-región sigue estando marcada por la centralización en la toma de decisiones, en el proceso 
de las políticas públicas, y por una marginal participación de las regiones en la discusión de los asuntos públicos en Chile.

Ahora bien, cabe preguntarse ¿como se relacionan los fenómenos de la descentralización, regionalización y políticas públicas? La respuesta estaría dada por la clasificación de los problemas públicos dentro de la agenda de gobierno. Para autores como Lahera (2002) y Asher (1992) las políticas surgen como respuestas a problemas públicos que son definidos y priorizados en una agenda de gobierno. Para ello deben converger intereses, tramas de poder y una diversidad de actores públicos y privados. En Chile, se observan rasgos característicos al interior de su realidad político institucional, que apuntan a la existencia de una separación entre el núcleo de poder central y las regiones, en la detección de necesidades públicas de carácter regional y en la formulación de políticas públicas, lo que genera un problema con dos dimensiones: una se vincula a la pertinencia y eventualmente de legitimidad en el origen de ciertas políticas emanadas del gobierno, situación que se sustenta en que la generación de políticas públicas en Chile mayoritariamente tiene su origen en la discusión que se genera a partir de actores que están territorialmente ubicados junto al núcleo del poder central como son los holdings empresariales, centros académicos, gobierno central, y partidos políticos.

La otra dimensión evidencia la falta de capacidad de las regiones para participar, definir y proponer iniciativas que puedan ser a lo menos consideradas como insumos para políticas públicas, máxime si estas atañen directamente su ámbito territorial. Tampoco se visualiza masa crítica regional activa e interesada en generar discusión al respecto, ni esfuerzos para generar esas condiciones.

Tal situación evidencia patrones institucionales y de negociación centralistas, aparejados con figuras institucionales como la de un intendente regional designado, que restaría legitimidad a una eventual gestación de políticas desde regiones. A eso se suma la no existencia de instancias de participación, ni de estructuras institucionales regionales para la construcción de políticas regionales (Podestá Arzubiaga, 2003).

Así el debate en torno al problema esbozado tiene otros alcances como la ausencia de modelos analíticos que sean capaces de dar miradas, congruentes, al fenómeno de las políticas públicas desde la perspectiva subnacional. Ello significa que aun faltan esfuerzos por adecuar metodologías y modelos que permitan explicar la realidad de un Estado altamente centralizado. 


\section{Las políticas públicas y los actores en democracia}

Las políticas públicas como disciplina implican unas reconsideraciones conceptuales y metodológicas para apreciar al gobierno y al Estado en acción, accionar que se observa en la construcción de políticas públicas (Subirats, 2001), reconsideraciones a las que no escapan los países latinoamericanos que se han caracterizado por la incorporación y búsqueda de nuevos enfoques y paradigmas que les ayuden a entender el funcionamiento del gobierno y el Estado.

En América Latina se había instalado tras la aparición de diversos gobiernos de corte militar, un enfoque relativamente cerrado y tecnocratizado de diseñar políticas públicas, situación que en las condiciones actuales se ve superado por los procesos de negociación, aprobación, rechazo y aplicación de políticas que coloca una serie de variables que no son consideradas en el enfoque tecnocrático (BID, 2005), porque pertenecen al ámbito de los gobiernos democráticos.

El informe sobre progreso económico y social del BID (Ipes) del 2005 se basó en una investigación para entender por que ciertos países de la región han tenido experiencias tan diferentes y muchas veces no muy efectivas con la aplicación de los modelos anglosajones, que habían operado con relativo éxito en dichos países. El enfoque actual, señalan, se debe basar en el análisis de los personajes o actores que intervienen en el proceso de construcción de políticas públicas (BID, 2005). Asimismo el español Subirats (2005), recalca la necesidad de reconocer a los actores que se movilizan en los escenarios políticos según sus preferencias e intereses y que buscan influir, condicionar, bloquear, o activar decisiones utilizando todo tipo de recursos. Para entregar su apoyo o rechazo despliegan una gama de recursos. En el caso de los actores privados pueden utilizar la publicidad, la financiación extra programática, prerrogativas de no invertir. En otras instancias emplean recursos de tipo político como manifestaciones, paros o movilizaciones y en otros casos o en conjunto recursos cognitivos, esto es, informes, investigaciones o artículos de expertos. La utilización de estos recursos puede ser de forma aislada o gradual o también de forma conjunta. En el caso de los actores públicos estos usan los mismos recursos con la diferencia que constan de un recurso que la sociedad civil no tiene: los recursos normativos. Los actores públicos constituidos por el gobierno y el aparato público tienen la capacidad de obligar, de acuerdo a los marcos regulatorios, a obedecer. Sus decisiones se pueden entender como decisiones soberanas aduciendo que corresponden al interés general, aunque 
nos sugiere Subirats, casi siempre son fruto de la interacción y negociación entre actores y de compromisos políticos.

La gama de actores que interviene en la formulación e implementación de las políticas públicas son variados, no obstante, según el estudio realizado el año 2005 por el BID, ${ }^{1}$ en el caso de América Latina los papeles estelares en la articulación de intereses en torno a las políticas públicas son tres. Los presidentes por su rol de representación democrática y sus amplias potestades, los partidos políticos le siguen por su papel como articuladores de relaciones entre el Legislativo y el Ejecutivo y como desagregadores de intereses en la aprobación de políticas. Aunque no son los únicos participantes en el proceso de construcción de políticas son quienes acaparan la atención en los países de la región. Finalmente los parlamentos, que aunque en la mayoría de los casos tienen una posición débil, son importantes. Ahora la pregunta que cabees: ¿cuál es el papel de los niveles subnacionales en la formulación de políticas públicas de carácter nacional?

\section{Políticas públicas y descentralización}

Uno de los requisitos de la política pública es que debe generarse en forma coordinada, no sólo con el resto de las políticas, además debe intentar coordinar e integrar la dimensión territorial, conformada por lo internacional, lo regional, provincial y comunal, o los niveles territoriales que existan. En ese contexto, las relaciones que se dan entre el Estado, en el marco de la generación de sus políticas con regiones, da cuenta de una relación compleja y atravesada por una serie de variables de tipo histórico, culturales y administrativas. Así lo expresa en su hipótesis Podestá Arzubiaga (2003). En su trabajo plantea que el aspecto más débil dentro del ciclo de políticas en Chile es el de formulación y evaluación de las políticas públicas. La afirmación apunta a que la relación Estado región se marca por una fragilidad en la conexión entre lo nacional y

\footnotetext{
${ }^{1}$ El estudio utilizó dos fuentes distintas de datos: la encuesta de opinión ejecutiva (Executive Opinión Survey) del informe sobre la competitividad mundial (Global Competitiveness Report) del Foro Económico Mundial, que abarca a más de 100 países y se ha venido publicando anualmente desde 1996, y una encuesta de capacidades del Estado realizada por el BID, que consultó la opinión de 150 expertos en 18 países latinoamericanos, entre ellos, analistas de políticas públicas, economistas, politólogos y ex ministros y altos funcionarios públicos, en cuanto a las capacidades del Estado con respecto a una serie de dimensiones identificadas como cruciales por Kent Weaver y Bert Rockman.
} 
lo regional, lo cual impide conseguir avances en la profundización de la democracia. Lo anterior se traduce en un obstáculo para que las regiones puedan alcanzar mayor crecimiento económico y desarrollo institucional sobre la base de la autonomía política. La relación entre políticas públicas y regiones es del tipo top down, donde los lineamientos del nivel central se trasladan, sin adecuaciones, a las regiones. Los efectos que se observan en este esquema es que las propuestas que se contienen en las políticas nacionales no consideran las particularidades del problema bajo la óptica regional. Otro aspecto observado que refuerza los efectos negativos de ese esquema es la falta de legitimación por parte de los actores regionales y la débil participación de la masa critica regional en la discusión, entendida como requisito para delimitar problemas públicos.

En suma, se puede afirmar que la relación entre las políticas públicas y las regiones es débil. Esta fragilidad se traduce en una serie de omisiones que se observan en el ciclo de políticas. La primera de ellas es la ausencia de un dispositivo para detectar las demandas y necesidades de la sociedad regional, elemento sustancial de la agenda pública. Podestá Azurbiaga nos señala que existe una disociación entre los que diseñan las políticas respecto de quienes son el público objetivo de las mismas. ${ }^{2}$ Lo anterior se traduce, según el autor, en que las decisiones y visiones contenidas en las políticas publicas, obedecen a criterios e intereses del nivel central. Así, el Estado, a pesar de impulsar un modelo de dispersión del poder legitimo, hacia los niveles subnacionales a través de una descentralización política y administrativa, no genera las condiciones para que al interior de las sociedades regionales se constituya una elite política ni una masa critica que cumpla esa función. Por otra parte, tampoco las regiones a través de sus universidades regionales, los partidos políticos o

\footnotetext{
${ }^{2}$ Un punto que no considera Podestá, en relación a su afirmación anterior, es que en esa disociación, o divorcio, como el le llama, igualmente se encuentra implícita una visón centralizada de la formulación de las políticas. Para él, el requisito de políticas que contribuyan a la descentralización, y que aporten a la competitividad de las unidades territoriales, pasa por que estas recojan información, conformada por las problemáticas regionales y se incorporen al proceso de formulación, que no se especifica en donde debe llevarse a cabo, en su trabajo. Otro aspecto que no considera dentro de sus conclusiones cuando hace alusión a crear un espacio de discusión sobre que políticas deben aplicarse al nivel territorial y que políticas fueran las apropiadas, para las regiones; olvida que la Locgar establece que las regiones pueden generar sus propios instrumentos de planificación para el desarrollo territorial. Se puede hablar que confiere, con ciertas restricciones de un instrumento que hace las veces de política pública regional, las estrategias de desarrollo, al cual los Gore no han sabido explotar su potencial, ni articular legitimidad por parte de los actores regionales, ni tampoco imbricar esa herramienta financiera con otras que se poseen.
} 
los gobiernos regionales, son capaces de generarlos, como tampoco hay evidencia de procesos de negociación ni de discusión política para la construcción de política pública en los espacios regionales.

Otro elemento del proceso de políticas públicas, que se observa débil en términos de legitimidad y correspondencia, es el levantamiento de información que se hace con fuentes documentales no regionales, sumándose a ello, la falta de actualización de la información, que generalmente es producto de diagnósticos sectoriales que realiza alguna oficina desconcentrada de algún ministerio. Finalmente en este punto, la información que se recoge por estas reparticiones centrales abusa del carácter cuantitativo, que si bien es un elemento de análisis gravitante, no da cuenta de las especificidades de la realidad regional, ello se podría mejorar con la incorporación de indicadores cualitativos que recojan variables como la identidad cultural, el contexto histórico y patrones de consumo entre otros.

Referente al tema de la evaluación de las políticas públicas, se observa la ausencia de una labor institucional regional respecto del impacto ex post en los territorios ${ }^{3}$ subnacionales. A pesar de contar con el recurso humano y las herramientas financieras, los gobiernos regionales se encuentran con la situación de que una evaluación para ser legítima debe atender a las metodologías y criterios establecidos por el nivel central. La paradoja, señala Podestá Azurbiaga (2003), es que mientras los criterios para evaluar los efectos positivos o negativos de las políticas nacionales en la región son definidos por el nivel central, las consecuencias se producen en la realidad regional. Junto a ello se suma la incapacidad de la sociedad regional de levantar propuestas para incidir en dicho proceso.

El desarrollo territorial en tanto, implica una serie de desafíos para los Gores que aparte de necesitar la indiscutible masa critica, académica, política e institucional, también necesita darle cuerpo y materialidad a las ideas surgidas de la elite regional, a través de los recursos financieros que no son descentralizados puestos que los Gores no cuentan con fondos propios importantes. Producto de lo anterior, las regiones deben negociar y discutir en desventaja con el nivel nacional-central (Santiago) durante el proceso de tramitación de la ley de presupuestos.

\footnotetext{
${ }^{3}$ Dicha información podría ser utilizada como datos empíricos de la conveniencia o no de la aplicación de políticas centralizadas a los niveles subnacionales y su grado de efectividad por áreas y el grado de legitimación por parte de los sectores involucrados. Su procesamiento y obtención seria insumo relevante para discutir sobre evidencia empírica de la necesidad (o no) de formular políticas regionales.
} 


\section{La descentralización en el nivel regional: elementos para entender la falta de participación de los Gore en la construcción y generación de políticas públicas}

Según los estudios realizados por la Subiere (Gobierno de Chile, 2001) existen una serie de nodos críticos que obstaculizan la profundización de la descentralización en Chile, y en particular para que los gobiernos regionales asuman su rol de articuladores del desarrollo en su territorio. Desde la lógica del Estado, gobierno significa, gobierno interior, una función que se remite a cuatro temáticas: ${ }^{4}$ orden público y seguridad ciudadana, coordinación, fiscalización o supervigilancia de los servicios públicos, prevención y atención de desastres, y extranjería. Cea Egaña ${ }^{5}$ en tanto nos dice que ejercer la función de gobierno implica realizar: la función política y ejecutiva, la función normativa, judicial y de control; y la función administrativa.

Desde la perspectiva politológica, gobierno significa ejercer el poder político legítimo del Estado y tener la capacidad, para diseñar y llevar a cabo proyectos de futuro. La pregunta que surge es: ¿cómo se relaciona una figura como la del gobierno regional que es en lo formal un órgano descentralizado territorialmente, pero que en la práctica su funcionamiento no dista del un órgano desconcentrado del poder central, que limitadamente ejerce una función de administración territorial con las prerrogativas del uso y ejercicio del poder legitimo?. La dicotomía se acrecienta ante lo que expresa el art. 16 de la Locgar sobre las funciones del Gore: "Elaborar y aprobar las políticas, planes y programas de desarrollo de la región, así como su proyecto de presupuesto, los que deberán ajustarse a la política nacional de desarrollo y al presupuesto nacional".

Siguiendo los argumentos de Boisier (2003:7), en ese artículo se contiene una función ejecutiva y política, que entrega al nivel regional la capacidad de formular sus propias políticas, según modelos de desarrollo acorde con las particularidades de su territorio. Tal situación implicaría una noción de con-

\footnotetext{
${ }^{4}$ Extraído de la Locgar 19.175.

${ }^{5}$ Para Cea Egaña (1999) las funciones que recaen en el intendente son incompatibles entre si, o a lo menos son conflictivas: las funciones ejecutivas donde le corresponde formular políticas de desarrollo de la región, ajustadas a los criterios centrales; presidente del consejo regional: esta instancia fue la fórmula para incorporar a la ciudadanía regional a la discusión de los temas de su territorio. En este consejo es el presidente con derecho a voz y a voto y en caso de empate dentro del consejo su voto es definitorio y representante del presidente de la República. En esa esfera le corresponde coordinar, supervigilar y fiscalizar el accionar de los servicios públicos que residen en la región de su jurisdicción.
} 
flicto entre el poder central y la región, siempre y cuando esta última tenga la potestad de contar con un núcleo de poder político, que le permita resolver tal diferencia con el nivel central en un contexto democrático. Tal situación, afirma el autor, implicaría la existencia de un régimen descentralizado como paradigma de reforma política del Estado en el que haya un régimen de una sociedad regional consensuada y autoreferida a su propio territorio.

Por otro lado los diagnósticos de la Subdere señalan que el rasgo que caracteriza la gestión regional es la asimetría entre las competencias, las funciones y las atribuciones que la ley confiere a los gobiernos regionales (Gobierno de Chile, 2001). Esa situación se refleja en la figura de los consejeros regionales (consejo regional) elegidos indirectamente ${ }^{6}$ por otros cuerpos colegiados (concejales agrupados por provincias) que en conjunto con el intendente conforman el gobierno regional, cuyo propósito es el desarrollo social, cultural y económico de la región. ${ }^{7}$ El hecho de que el intendente tiene la iniciativa en casi todos los temas que trata el consejo es una circunstancia que entraña una participación menor del consejo en la administración efectiva de la región.

En lo que respecta a las herramientas financieras que poseen los

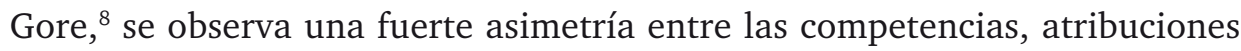

\footnotetext{
${ }^{6}$ En relación al mecanismo de generación de esta instancia representativa, que pasa por la elección de los consejeros a través de los concejales agrupados por provincia, se observa la operación de repartición de cuotas de poder partidista que se traducen en que se pierde la visión integrada de la región como sistema, más bien el accionar de los consejeros que deben responder a criterios partidistas, trae como consecuencia el apoyo a iniciativas parciales que buscan beneficiar a ciertas localidades en búsqueda de capital electoral, subordinando el interés general de la región a intereses mediáticos perdiendo el proyecto de unidad regional su propia identidad (Gobierno de Chile, 2001). Por otra parte la ausencia de mecanismos de control social y la priorización del presidente del consejo, en este caso el intendente, de las relaciones con el presidente de la República, en detrimento de la visión regional agudiza tensiones y cuestiona la legitimidad de la máxima autoridad regional.

${ }^{7}$ Ley no 19.175 (Orgánica Constitucional sobre Gobierno y Administración Regional).

${ }^{8}$ En relación a las Seremis se detectó por la Subdere que estos órganos presentan una serie de problemas en aspectos técnicos, normativos y en la calidad y cantidad del recurso humano y financiero para llevar a cabo su gestión. En juicio de los autores de ese diagnóstico, la desconcentración es más formal que efectiva. Prosiguen señalando que varias de las Seremis no tienen un control ni potestad completa sobre los servicios públicos enmarcados en su accionar. Para Boisier la instalación de esta repartición del gobierno central, a partir de las recomendaciones de la Conara, no se ha considerado las especificidades propias de cada región. En la práctica ello se ha traducido, según Boisier, en la instalación de Seremis que no tienen incidencia en el desarrollo productivo de una región determinada es un factor de doblamiento innecesario del nivel regional. También se destaca como aspecto negativo la doble dependencia de las Seremis que le impiden responder a los
} 
y funciones, dado que las últimas no son las suficientes para procurar el desarrollo territorial. Boisier enuncia la interrogante de si es posible pensar una descentralización sin recursos financieros. El diagnóstico Subdere menciona que no son suficientes los recursos financieros para cumplir con las tareas que la ley les confiere. El FNDR, en tanto, se concibe como una herramienta subsidiaria orientada a sectores, no obstante faltan elementos para que se constituya en un instrumento que contribuya al desarrollo regional, atacando la desigualdad territorial. Se suma además que el FNDR no cuenta con una base participativa de los actores regionales para su conformación; además de la poca interacción con la estrategia regional de desarrollo y los demás instrumentos de planificación del desarrollo regional (Gobierno de Chile, 2000:10). Se expresa que el presupuesto por gastos de funcionamiento, no es capaz de dar cuenta de todas las tareas que el Gore debe asumir en las áreas de fomento productivo, desarrollo social y ordenamiento espacial. Prosigue al respecto aludiendo que el programa de gastos para el funcionamiento de esa institución no ha superado históricamente el 7\% del presupuesto general (Subiere, 2001:52).

Tabla 1

Participación porcentual de los niveles territoriales en las finanzas públicas

\begin{tabular}{|c|c|c|c|c|c|c|c|c|}
\hline \multirow[t]{2}{*}{ Año } & \multicolumn{4}{|c|}{$\begin{array}{l}\text { Participación en el presupuesto } \\
\text { de ingreso público }\end{array}$} & \multicolumn{4}{|c|}{$\begin{array}{l}\text { Participación en el presupuesto } \\
\text { de gasto público }\end{array}$} \\
\hline & Nacional & Regional & Comunal & Total & Nacional & Regional & Comunal & Total \\
\hline 1990 & 93,5 & 0,1 & 6,4 & 100 & 87,3 & 1,3 & 11,4 & 100 \\
\hline 1991 & 93,2 & 0,2 & 6,5 & 100 & 87,1 & 1,4 & 11,5 & 100 \\
\hline 1992 & 93,1 & 0,2 & 6,7 & 100 & 86,1 & 1,5 & 12,4 & 100 \\
\hline 1993 & 92,9 & 0,2 & 7,0 & 100 & 85,7 & 2,6 & 11,7 & 100 \\
\hline 1994 & 93,0 & 0,2 & 6,8 & 100 & 84,3 & 2,8 & 12,9 & 100 \\
\hline 1995 & 92,6 & 0,1 & 7,3 & 100 & 84,0 & 2,9 & 13,1 & 100 \\
\hline 1996 & 92,2 & 0,3 & 7,5 & 100 & 82,3 & 3,4 & 13,7 & 100 \\
\hline 1997 & 92,1 & 0,2 & 7,6 & 100 & 81,7 & 4,1 & 14,2 & 100 \\
\hline 1998 & 91,6 & 0,2 & 8,1 & 100 & 81,0 & 4,8 & 14,2 & 100 \\
\hline
\end{tabular}

Fuente: Gobierno de Chile, 2000.

requerimientos que implica procurar el desarrollo territorial regional y la dependencia financiera de sus recursos por parte del nivel central, refuerza la situación anterior. 
Tabla 2

Presupuestos de los gobiernos regionales hasta el año 2000 (base de cálculo: 151.450.061 m de pesos)

\begin{tabular}{|lrlr|}
\hline Ingresos FNDR & $\%$ & Gastos FNDR & \multicolumn{1}{c|}{$\%$} \\
\hline Ingresos de operación & 0,2 & Inversión real & 99,1 \\
Transferencias & 85,1 & Saldo inicial de caja & 0,8 \\
Otros ingresos & 0,0 & Total gastos FNDR & 100 \\
Aporte fiscal & 13,7 & Funcionamiento & \\
Saldo inicial de caja & 0,9 & Gastos en personal & 72,8 \\
Total FNDR & 100,0 & Bienes y servicios de consumo & 17,2 \\
Funcionamiento & & Transferencias corrientes & 8,2 \\
Aporte fiscal & 99,9 & Inversión real & 1,6 \\
Saldo inicial de caja & 0,1 & Otros gastos & 0,3 \\
Total funcionamiento & 100,0 & Total funcionamiento & 100,0 \\
Presupuesto total FNDR & & & 93,4 \\
Presupuesto total funcionamiento & & & 6,6 \\
Total presupuesto gobiernos regionales & & & 100,0 \\
Presupuesto gobiernos regionales/presupuesto de al nación & 2,0 \\
\hline
\end{tabular}

Fuente: Ley de Presupuestos del sector público año 2000.

Una de las debilidades que se observa a los fondos regionales es el alto grado de centralización en su asignación, que condiciona el programa de inversiones del FNDR. El contra argumento desde la centralidad alude a la inexistencia de ingresos propios por parte de las regiones. Así la totalidad de los recursos son derivados desde el nivel central por medio de la Ley de Presupuestos, cuya asignación tiene como criterio orientador la estabilidad macro presupuestaria (Subdere, 2001:53).

En la actualidad el nivel central sostiene que aproximadamente un $50 \%$ de la inversión en el sector público es decidido por las regiones. No obstante, dando una mirada desde la globalidad de las finanzas públicas la inversión pública constituye cerca del 18\% de la inversión nacional, por tanto, los niveles subnacionales deciden, usando un concepto relativo de decisión, un 9\% de la inversión nacional. Así, cada región en específico decidiría (usando como base una media simple) sobre un $0,7 \%$ de la inversión nacional (Boisier, 2003). 
Finalmente en relación a las estrategias de desarrollo regional ${ }^{9}$ como instrumento de planificación regional las conclusiones apuntan a que deben constituirse en un instrumento efectivo para la gestión. Para lograr ese cometido la división de evaluación y cuentas públicas de la Subdere (Gobierno de Chile, 2000) recomienda modificar su estructura y su forma de generación. El establecimiento de contenidos estándares y necesarios, mayor precisión y la incorporación de dispositivos que aseguren la participación de los actores regionales tanto del sector público como privado. En tanto la legitimación se instala como un elemento central para darle sustentabilidad a dicho instrumento, como mecanismo de socialización y aceptación.

\section{Descripción del caso: la Política Nacional de Acuicultura y la participación del Gore de Los Lagos y SalmonChile en su definición}

En la actualidad Chile es el segundo productor de Salmones a nivel mundial. El sector salmonicultor se ha convertido en el cuarto sector exportador nacional; en relación al crecimiento y desarrollo productivo a nivel regional la acuicultura se ha transformado en un sector estratégico que es el motor productivo que moviliza la actividad económica de la región de Los Lagos. Se constituye en la principal fuente de empleos y de exportaciones. En materia de empleos en la X Región son unos 45 mil los que genera en forma directa e indirecta. En cuanto a las exportaciones, este sector para el 2004 genero el $80 \%$ de las exportaciones de la región de Los Lagos, en términos nacionales alcanzó un 5,5\% de todo el producto de lo que Chile exporta (Pérez Guerra, 2003:2). En la actualidad Chile controla el 35\% del mercado mundial, mientras que Noruega concentra el 37\%. La razón de este crecimiento y valoración exponencial radica en las cifras que han obtenido como generadoras de divisas y aportes al PIB regional y nacional (Ministerio de Economía, 2002).

\footnotetext{
${ }^{9}$ La estrategia regional de desarrollo fue incorporada al inicio del proceso de democratización en 1990 y formalizado a través de la Ley Orgánica Constitucional sobre Administración y Gobierno Regional en 1993. Son elaboradas por las secretarías regionales de Planificación en cada región y el gobierno regional debe decidir sobre la pertinencia de dicho instrumento. El mecanismo para resolver la aprobación o no de la propuesta es a través de un proceso, no muy claro, de discusión interinstitucional y participación ciudadana (Ministerio del Interior, 2002:89).
} 
Tabla 3

Principales exportaciones de alimentos - año 2005

\begin{tabular}{|lr|}
\hline Producto & US\$ MM \\
\hline Harina de pescado & 445,6 \\
Salmón y truchas & $1.657,5$ \\
Moluscos y crustáceos & 281,6 \\
Conservas de pescado & 147,4 \\
Puré y jugos de tomate & 54,8 \\
Jugos en polvo & 88,6 \\
Carnes de cerdo & 295,7 \\
Vino & 882,5 \\
Fruta seca & $2.128,9$ \\
Fruta deshidratada & 181,4 \\
Conserva de frutas & 83,6 \\
Jugos de frutas & 122,0 \\
Fruta congelada sin azúcar & 116,5 \\
Total & $7.224,1$ \\
\hline
\end{tabular}

Fuentes: Banco Central de Chile e Informe Económico de la Salmonicultura, 2005.

La actividad de la acuicultura y específicamente la salmonicultura se ha concentrado en la X Región. Según cifras de SalmonChile, en el año 2004 un $84 \%$ de la producción se originó en la región de Los Lagos. Por tanto una actividad que exporta más de US\$ 1.700 millones ha generado efectos significativos sobre la economía regional. Según este informe, que utiliza datos del Banco Central, señala que el valor agregado bruto de la industria se sitúo en torno a los US\$ 1.200 millones, equivalente a casi un punto del PIB (Informe Económico de la Salmonicultura, 2005:14).

La relación existente entre el desempeño económico regional (Inacer) para la región de Los Lagos y las exportaciones de salmón junto con el crecimiento del PIB regional en relación con el de otras regiones se presenta en el grafico 1. El gráfico 2 muestra la evolución del PIB de la X Región en relación al PIB nacional, el PIB de las regiones del centro sur (VI a XII Regiones) y el PIB de las regiones no mineras (III a XII Regiones) para el período 1996-2005. Se aprecia que en los últimos 10 años, el PIB de la X Región creció entre un 
10\% y un 15\% más que las demás áreas geográficas señaladas, en particular respecto del PIB de las regiones no mineras (regiones VI-XII).

\section{La Política Nacional Acuícola (PNA)}

El estudio realizado por el Ministerio de Economía en el marco del Programa de Prospectiva Tecnológica encargado ad-portas a la discusión de la Política Nacional Acuícola (PNA) concluía que en términos institucionales y regulatorios la actividad acuícola es una de las más reguladas del país en cuanto al acceso. Prosigue señalando que la tramitación de solicitudes y autorizaciones eran complejas, lentas y engorrosas (Subpesca, 2003a). Junto con lo anterior los limitados recursos humanos y financieros se traducían en problemas de eficiencia y oportunidad de las fiscalizaciones y controles, contribuían a la ausencia de un panorama de certidumbre y estabilidad para el desarrollo del sector, donde privados desistían de invertir por lo complicado del acceso.

\section{Gráfico 1}

PIB de la X Región en relacón a otras áreas geográficas (Î́ndice 1996=100)

\section{Período 1996-2003}

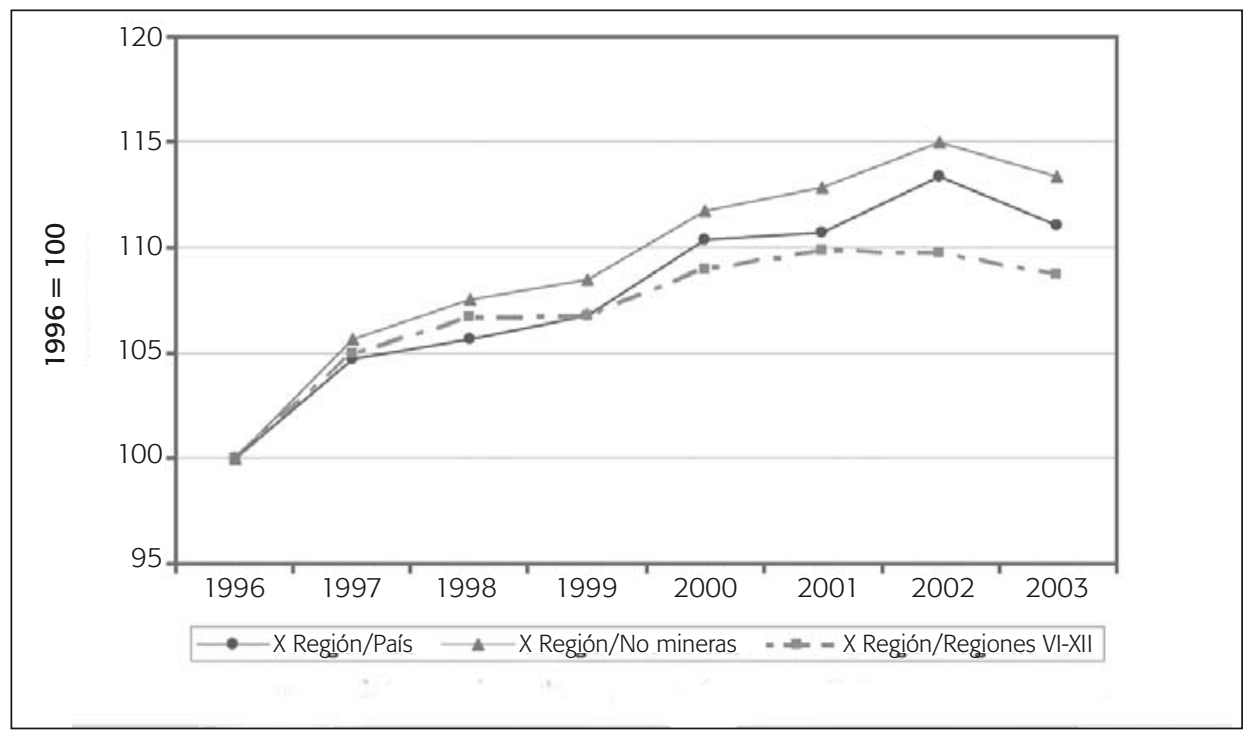


Gráfico 2

Relación entre INACER X Región y exportaciones de salmón Período Marzo 1996 - Marzo 2005

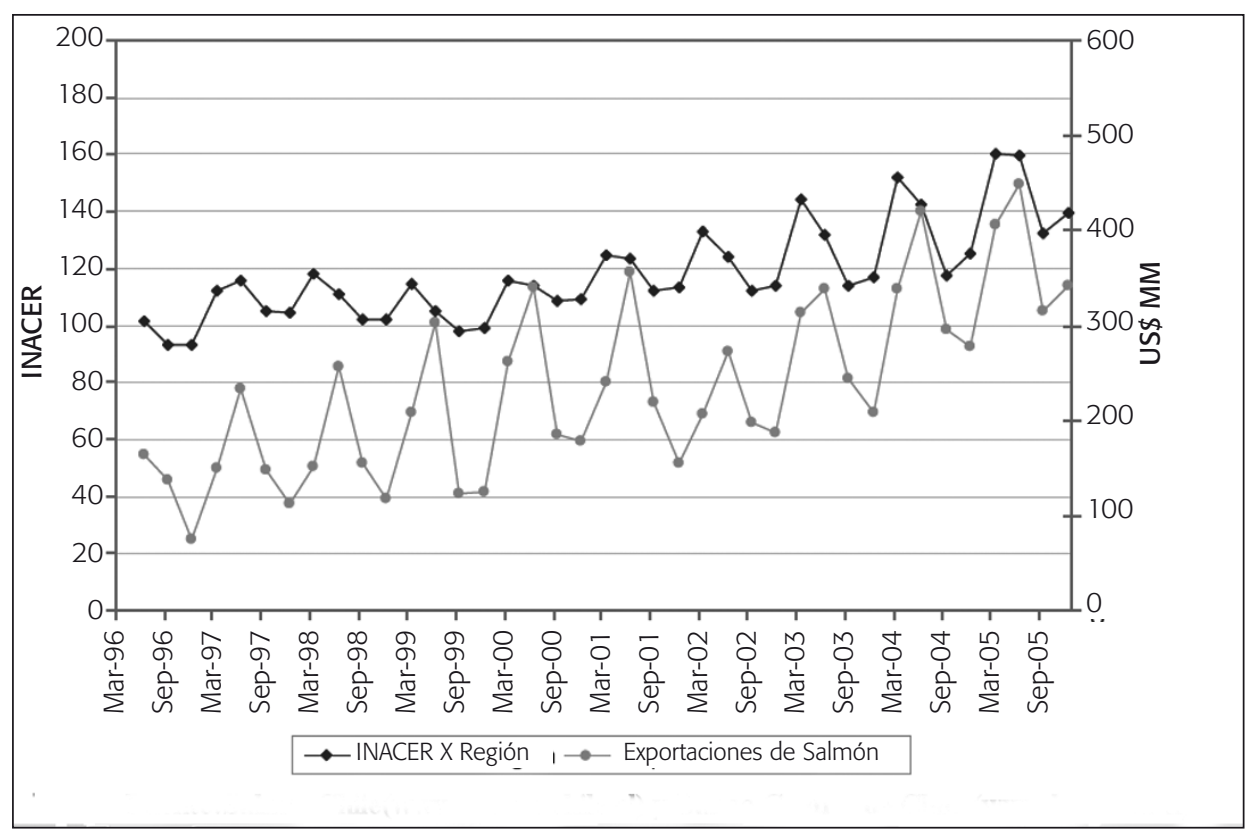

Los aspectos que aborda esta política son: la sustentabilidad económica y medio ambiental, protección de la masa biomarina, equidad para acceder a la actividad, directrices para el fomento de la capacitación y la investigación, y readecuación de la institucionalidad pública y el marco regulatorio (Subpesca, 2003a). Para los sectores público y privado, establece un marco tanto valórico como regulatorio de referencia para sus actuaciones. Para la industria establece como desafío enfrentar el desarrollo del sector a través del cuidado al medio ambiente en un contexto de economía social de mercado, que contextualiza el quehacer económico del país, y de la integración económica y política que se enmarca en la globalización. Para el sector público se establece como desafío, definir sus competencias y realizar su función de manera efectiva y ágil, además, como garante del bien común, se le establece el imperativo de velar por el cuidado del medio ambiente, a través de normas consistentes, que hagan compatibles el máximo crecimiento económico en armonía con el medio ambiente, además de cuidar el libre acceso a la actividad para todos aquellos que deseen y puedan hacerlo acorde a sus capacidades (Rivera, 
2003). Los aspectos antes señalados sirven como marco para la instauración de principios ${ }^{10}$ que deben orientar el accionar público y privado de acuerdo a lo establecido por la PNA. ${ }^{11}$

Para Rivera la promulgación de la PNA se atiende por la sostenida relevancia del sector acuícola y en especifico de la salmonicultura, la articulación de este crecimiento y el impacto en otros sectores como la geografía nacional, la capacidad de empleo y su contribución al PIB nacional desde el nivel regional, gatillaron la decisión del aparato gubernamental de impulsar una política específica para ese sector (Rivera, 2003).

El objetivo de la PNA se traduce en: "Promover el máximo nivel posible de crecimiento económico de la acuicultura chilena en el tiempo, en un marco de sustentabilidad medio ambiental y de igualdad de oportunidades en el acceso" (Subpesca, 2003a). Esto debe interpretarse como que el crecimiento económico es el elemento que articula a los otros dos elementos, puesto que sustentabilidad en su acepción amplia, mas la equidad son los soportes que guían dicho crecimiento. En tanto sustentabilidad medio ambiental apunta al intento de homologar estándares que son utilizados por países desarrollados y finalmente equidad, que según la autoridad sería la base para el aprovechamiento de las potencialidades por el sector y el país, toda vez que integra la igualdad de oportunidades para poder ejercer la actividad acuícola con fines lucrativos, considerando que el recurso utilizado para su producción son aguas marinas que pertenecen al Estado chileno.

\section{Aspectos metodológicos}

La metodología de trabajo consideró la aplicación de entrevistas en profundidad a los actores clave del sistema, y el análisis documental previo para preparar las entrevistas. En efecto los actores considerados fueron los siguientes.

\footnotetext{
${ }^{10}$ Subpesca (2003b) comunicado aparecido en el portal de esa institución tras la promulgación de la PNA el 3 de agosto el 2003 hecha por el presidente de ese periodo, Ricardo Lagos Escobar.

${ }^{11}$ Estos principios son: sustentabilidad en el tiempo, articulación y cohesión de la política de este sector con otras de nivel productivo y sectorial; la corresponsabilidad pública y privada como base de la planificación estratégica del sector; el mejoramiento en el acceso que implique una diferenciación entre la actividad industrial y artesanal; la competencia de la institucionalidad en términos de oportunidad y experticia; la incorporación de la investigación científica y la innovación tecnológica como principios que orientarían un accionar más competitivo buscando soluciones y formulas que propendan al desarrollo del sector.
} 
Definición de actores clave: los actores involucrados en la formulación de la PNA fueron de carácter público y de carácter privado, entre los primeros existieron funcionarios del nivel central y del nivel regional, destacándose a nivel central el jefe de la División de Acuicultura de la Subsecretaria de Pesca, que coordinó la discusión y tramitación de la Política Nacional Acuícola. Este cargo tiene un fuerte componente técnico (el responsable es biólogo marino) por la naturaleza de las materias y además un componente político, puesto que debe coordinar, negociar, consensuar y tratar de legitimar las acciones gubernamentales del nivel central, sobre el área económica productiva de su competencia.

El actual diputado del distrito 57, presidente de la Comisión de Pesca y Acuicultura de la Cámara y ex intendente regional es otro actor clave dentro de la discusión de la PNA. Cabe señalar que en ese entonces el ex intendente relevó el tema de la pesca y la acuicultura poniendo énfasis en los sectores artesanales, y valorando los aportes de la industria por el impacto en términos de crecimiento que esta genera en la región.

Se consideró también la instancia del consejo regional, específicamente la Comisión de Pesca y Acuicultura, en consideración a que esta es una instancia de representación de los intereses de una de las regiones más importante en materia de pesca y acuicultura. Una de las principales dificultades fue que esta instancia respondiera a los cuestionarios demostrando evidente falta de interés, como de igual manera ocurrió en la tramitación de la PNA.

El último actor dentro de este grupo de estudio referente a la participación de la región de Los Lagos en la PNA fue el gremio SalmonChile. Este gremio es el más importante en términos de presencia corporativa en la región, se entrevistó a su gerente general que es el encargado de articular, promover, dialogar y gestionar las acciones del gremio tanto en el nivel político institucional, como en el área productiva. Tuvo una participación activa en el diagnostico de la actividad que realizo la división de prospectiva tecnológica del Ministerio de Economía que sirvió de insumo para la PNA.

El grupo de control estuvo determinado por el gobierno regional en las actuales autoridades; consejo regional específicamente la Comisión de Pesca y Acuicultura y el intendente regional con quienes se contrastó la información obtenida a través de las entrevistas, en la idea de identificar la presencia o no de las variables consideradas en las hipótesis, relacionadas con la incapacidad del gobierno regional para formular sus propias políticas de manera consistente.

Aplicación de instrumento: se aplicó una entrevista semiestructurada en profundidad. Se construyeron las categorías de análisis con el cuidado que en ellas se pudieran incluir palabras, frases o conceptos que permitan encontrar la presencia o ausencia de las variables a medir. Según palabras de Doña (2006:3) 
las categorías corresponden a grupos significativos que dan pie a la clasificación en las unidades de registro. Se incorporaron las recomendaciones de Lécuyer citado por Colle (2005), sobre el modelo cerrado donde las categorías están predeterminadas por el investigador desde el comienzo, a partir de cuerpos teóricos desde los cuales se basa el estudio para plantear las hipótesis.

\begin{tabular}{|ll|}
\hline Categorías de análisis & Hipótesis 1 \\
\hline Potestades institucionales del Gore de Los Lagos para formular políticas & Hipótesis 1 \\
Participación marginal en el diseño y formulación de la PNA & Hipótesis 2 \\
(Ausencia) Mecanismos que sirvan para procesar problemáticas regionales & Hipótesis 2 \\
Insumos para políticas públicas de nivel regional o nacional & Hipótesis 3 \\
Ingerencia del sector acuícola como actor regional en el proceso de & Hipótesis 3 \\
\hline definición de la PNA & \\
(Contribuyo, más) Modernización de la institucionalidad acuícola & \\
\hline
\end{tabular}

Posteriormente se aplicó un análisis con diseño intensivo transversal donde se procedió a la búsqueda en términos de importancia, significación y relaciones de los datos obtenidos. De esta forma se rastreó en las entrevistas las afirmaciones relacionadas con las preguntas de investigación y las hipótesis. Las palabras y conceptos se enlazaron con los temas y con las ideas en la intención de que sirvieran para ir dando respuesta a las hipótesis. Cabe relevar que este no fue un análisis cuantitativo y no recurrió a procedimientos de muestreo. Los significados e inferencias se lograron analizando ${ }^{12}$ (Piñuel, 2006): relaciones, oposiciones y considerando el contexto. Se revisaron las discrepancias o coincidencias de lo declarado por los sujetos entrevistados, que en el contexto de la discusión de la PNA fueron actores claves, y para el contexto regional también.

\section{Presentación de los resultados}

Referente a la hipótesis 1: señalaba que el gobierno regional de Los Lagos no utiliza sus potestades para concebir políticas públicas a nivel regional según

\footnotetext{
${ }^{12}$ Ejemplo de este tipo de diseño es el estudio de Hoilsti Body e North (1965) quienes analizaron todo el material periodístico emanado de los actores clave durante la crisis de los mísiles cubanos en 1962 y la evaluaron según la teoría de las actitudes de Osgoods. Mediante sus inferencias propusieron un modelo de interdependencias dinámico de la Guerra Fría en situaciones de crisis.
} 
se lo confiere la LOC no 19.175 y que participó marginalmente en el diseño y formulación de la Política Nacional de Acuicultura. Se obtuvo de las afirmaciones contenidas en las respuestas del ex intendente, respecto de las potestades constitucionales para formular políticas regionales, hacían alusión a programas de inversión e iniciativas por áreas productivas y sociales, pero que no guardan relación con políticas regionales a largo plazo, según una política direccionada, legitimada y definida técnica y democráticamente. Sus respuestas denotan que no conocía tales potestades.

En tanto al ser consultado sobre si participó el Gore de Los Lagos en la discusión y definición de la PNA durante su mandato, la respuesta apuntó a que no participaron activamente en la discusión. La razón que argumenta fue que el nivel central, en este caso Subpesca, ya tenia definida su agenda de trabajo. No obstante, como se revela en las siguientes entrevistas la no participación se explica por otros factores.

En tanto el jefe del Dpto. de Acuicultura de la Subpesca, quien coordinó la discusión y tramitación de la PNA afirma:

No. (R: 13) Durante el proceso de elaboración y discusión de las propuestas se invitó a participar a todos los gobiernos regionales que tenían actividades de acuicultura en su jurisdicción. (R: 11) hubo escasa participación activa por parte de la mayoría de ellos, incluyendo al de la región de Los Lagos (R: 11)

Finalmente el gerente general de SalmonChile, consultado sobre la participación del Gore de Los Lagos en la discusión y tramitación de la PNA señala:

No recuerdo que haya habido alguna conversación con la gente del gobierno regional de Los Lagos sobre la Política Nacional de Acuicultura (I: 4) Recuerdo que esto lo vimos con el nivel central, por que de hecho se veía ahí en el contexto de la acuicultura y de los gremios acuícolas con la Subsecretaria de Pesca y ahí se fue desarrollando la discusión al respecto (I: 4)

En cuanto a la hipótesis 2: que afirmaba que el gobierno regional de Los Lagos no cuenta con mecanismos, que sirvan para procesar problemáticas regionales que luego se traduzcan en insumos para políticas públicas regionales, ni para las políticas de nivel central que aporten a una visión de crecimiento y desarrollo desde la región al resto del país, se tuvo que el ex intendente señalo: "Se le puede sacar mayor provecho a estos instrumentos pasando de la focalización a la priorización". La respuesta anterior denota que aun son 
muy generales y que por cierto no explica cuales son. Sigue posteriormente afirmando que "Se plantea como desafío alcanzar mayores niveles de legitimidad" (refiriéndose a la ERD) y que "debe contener elementos vinculantes con la ciudadanía o sociedad regional".

En tanto el actual intendente señala que: "las ERD tradicionalmente han carecido de un poder vinculante y de cierta legitimidad social" para luego concluir: "implementar una potente batería de instrumentos y apalancar una mayor cantidad de recursos para apoyar y calificar el recurso humano en torno a la vocación productiva de la región en torno al desarrollo de las PYME". En la primera afirmación refuerza lo dicho por el ex intendente y posteriormente señalar que implementarán una batería de instrumentos, lo cual implica que aun no existen mecanismos con las características antes señaladas. Como medida remedial y luego de 13 años de funcionamiento anuncia la instauración de la División de Planificación y Ordenamiento Territorial y señala: "La creación de la tercera División de Planificación y Ordenamiento Territorial del Gore, implementada durante esta gestión, viene a subsanar esta debilidad, al generar el soporte técnico y humano que permita sistematizar toda al información entrópica y transformarla en información inteligente al servicio del desarrollo regional (J: 5)".

La consejera por su parte afirma:

Los mecanismos de participación efectivamente pueden y deben ser mejorados (S: 12) Tengo la sensación que la gente participa poco y en cada localidad son siempre los mismos (S: 11) Desincentivamos la participación si no hacemos un efectivo seguimiento de los proyectos para verificar que estos se implementen tal como fueron aprobados y posteriormente su funcionamiento sea compatible con lo aprobado (S: 13) Mientras no se acote convenientemente la ERD, se puede justificar un cambio de rumbo severo (S: 25)

Por lo anterior es posible señalar que no existen mecanismos que sirvan para procesar problemáticas regionales a excepción de las ERD, pero con la limitante de su débil legitimidad. Este es un elemento fundamental, puesto que un rasgo que caracterizaría un mecanismo así, sería el levantamiento de información (necesidades y requerimientos por parte de los distintos actores regionales incluido la ciudadanía). Se observa entonces la ausencia de esta variable en la muestra recogida. Se releva el antecedente de la creación de una división administrativa que seria el primer esfuerzo estructurado para pensar desde la perspectiva regional el crecimiento de la región. 
Por ultimo en relación a la hipótesis 3: que sostenía que un actor relevante en la región de Los Lagos como generador de crecimiento económico, específicamente el sector acuícola (SalmonChile), es capaz de influir positivamente en el proceso de políticas públicas, ayudando a modernizar la institucionalidad y las normas, en el ámbito de su interés. Se puede establecer, de acuerdo a lo respondido por el jefe del Dpto. de Acuicultura de la Subpesca, que en cuanto a la participación del gremio SalmonChile en la discusión de la PNA y los aportes entregados por este gremio fueron relevantes:

En general si, junto con la Asociación de Productores de Ostras y Ostiones de Chile (Apooch), Asociación de Mitilicultores de Chiloé y Conapach (R: 9) Fueron los que más se involucraron tanto en la fase de diagnóstico como en las propuesta de política. (R: 9) En términos generales participaron activamente en las presentaciones y discusiones realizadas con los(as) representantes del sector privado. (R: 10)

Finalmente el gerente de SalmonChile al ser preguntado sobre su papel activo en la discusión, en su calidad de gremio y sobre los elementos que lograron añadir en la política respondió:

Recuerdo que esto lo vimos con el nivel central, por que de hecho se veía ahí en el contexto de la acuicultura y de los gremios acuícolas con la subsecretaria de pesca y ahí se fue desarrollando la discusión al respecto. Bis (I: 4) Yo creo que para nosotros una cosa que es esencial en la operación yo creo que el tema de la simplificación de lo tramites y el tema medio ambiental para hacer mas competitivo el sector, el tema de la innovación a través de Intesal (I: 6) evitar que hayan áreas que produzcan que se vaya quedando trancados precisamente por que toda la parte administrativa se hace un poco tediosa por que son muchas instancias que participan (I: 6)

Para SalmonChile, modernizar la institucionalidad y los trámites era un punto crucial. Eso se cumplió con la agilización de los trámites de concesión y la conformación de la comisión nacional acuícola que trabaja de manera permanente en ese y otros temas. El tema medio ambiental también era de interés para SalmonChile en términos de cómo aumentar la trazabilidad de los productos. Finalmente en términos de innovación Intesal fue una instancia creada para revertir ese punto y seguir creciendo.

Un punto que el representante de SalmonChile señalo que no se incorporó, fue la creación de una agencia, para la promoción de la actividad con líneas de apoyo específico. Algo así como el Indap de la acuicultura a toda escala. 


\section{A modo de conclusiones}

Los elementos que se consideraron para definir el objeto de estudio de la investigación fue la cuestión territorial. Ese criterio se adoptó en virtud de que la región de Los Lagos es la localización donde la actividad acuícola produce sus mayores impactos en términos de generación de empleos, contribución al PIB regional y también al tema medio ambiental. Otro rasgo que se quiso abordar fue la capacidad de participar en la formulación de políticas de tipo nacional, del gobierno regional de Los Lagos que era el actor regional institucional, al que por cuestiones de crecimiento debería haberle importado la discusión de una política nacional que impactaba su territorio. Eso fue considerado una oportunidad en el estudio para establecer algunas generalizaciones respecto de la capacidad de ese órgano para actuar de manera articulada velando por el crecimiento de su territorio en un contexto de descentralización.

Complementariamente se quiso comparar la participación en el campo de las políticas públicas de dos actores regionales: El gobierno regional de Los Lagos como actor institucional y SalmonChile como actor privado, que por la localización territorial de su actividad, principalmente en la región de Los Lagos, hacía que la PNA fuera de especial interés para ellos, por los efectos que podía causar en su sector. Teniendo claro ese contexto, se trató de verificar que actor regional, en este caso entre SalmonChile y el gobierno regional de Los Lagos, logró participar y aportar mas elementos en la discusión y tramitación de la Política Nacional Acuícola.

De esta forma es posible establecer algunas conclusiones que el estudio logró evidenciar, entre estas se puede señalar:

v que el gobierno regional de Los Lagos tuvo una participación menor en la discusión y tramitación de la PNA. Esta afirmación se funda en las respuestas entregadas por el encargado del gobierno central perteneciente a la Subsecretaria de Pesca que fue quien coordinó la discusión de la PNA. Este actor responde de manera certera, que a pesar de las invitaciones que se hicieron a las diversas instancias involucradas: universidades, gobiernos regionales y otros sectores su participación fue escasa. Eso es reforzado por la consejera regional que es miembro de la Comisión Pesca y Acuicultura del Consejo Regional, que señala que el tema nunca se vio como Gore, y tampoco recibió noticias del Seremi de Economía, que por sector debería haber coordinado alguna acción al respecto. El triángulo lo cierra el ex intendente quién afirma que el sector acuícola, como autoridad lo visualizaba como de gran importancia para el crecimiento de la región, y no participaron de la discusión; 
- el Gore en ese entonces; señaló, estaba en el tema en la práctica (en alusión a un programa para la construcción de infraestructura en la localidad de Carelmapu para los pescadores artesanales). Por tanto se observó, de acuerdo a los antecedentes que se procesaron, que el gobierno regional de Los Lagos no logró incorporar ningún aporte desde la perspectiva regional a la discusión, que si contó con los aportes, participación o interés de los ecologistas, artesanales y gremios acuícolas de mayor tamaño;

v que SalmonChile, considerado como actor regional, logro una participación más activa incorporando elementos que eran de su interés. En este punto se da por sentado que el aporte en términos de participación lo efectúa en virtud de sus intereses de índole económico; ello refuerza la trama planteada en la base teórica que los actores se movilizan en torno a los intereses que desean defender. A diferencia del Gore de Los Lagos que debió haber desplegado sus atribuciones y capacidades en pos de aportar, cuidando los intereses de la región desde la mirada institucional.

Dentro de los elementos que SalmonChile logró incorporar en acuerdo con el gobierno central, estaba el tema de los trámites, para hacerlos mas expeditos y no perder oportunidades de negocio ni competitividad, sin que eso significara descuidar aspectos como los medio ambientales. Cuestión defendida por el nivel central y grupos ecologistas. En materia de producción limpia, se establecieron algunos compromisos para una comisión de trabajo específica que se ocupara del tema donde participa la autoridad central (Subpesca), la autoridad sanitaria, las instituciones medio ambientales tanto del nivel central como regional, en este caso Conama y Corema.

Como punto enfrentado con la compatibilidad de la actividad acuícola y la turística está el tema del manejo del borde costero, que implica que los gobiernos regionales estén en capacidades de dialogo con las Coremas para establecer zonas autorizadas para concesiones acuícolas, en armonía tanto desde el punto de vista ambiental, en términos de poluciones como del cuidado de los paisajes como patrimonio natural, y potencial área económica de mayor presencia.

Estos puntos, señala el entrevistado de la Subsecretaria de Pesca fueron discutidos por los gremios artesanales SalmonChile y Productores de Mitílidos. Por su parte, el gerente general de SalmonChile, señaló que esos puntos se trabajaron durante la discusión de la Política Nacional Acuícola. Aun más, señala que el gobierno central no incorporó otro tema que era importante para ellos, que decía relación con la instauración de un órgano específico que ayude al fomento de la actividad. Esto se traducía en una agencia ocupada de potenciar la industria y abrir nuevos mercados. En este punto se podría com- 
partir o no que esta instancia corresponda o no al Estado, pero evidencia la participación de SalmonChile en la discusión.

Se afirma en virtud de lo anterior, que en materia de descentralización y considerando el caso del Gore de Los Lagos, existen debilidades en materia de planificación regional como insumo para un crecimiento armónico, que propenda al fortalecimiento de la participación como correlato de la democracia representativa y de la descentralización como modelo de dispersión del poder político, y segundo de la competitividad, en la medida que se aprovechan de manera organizada y planificada las potencialidades de cada territorio, ante las condiciones que pone la economía de mercado a países pequeños como Chile.

Estas afirmaciones se realizan a propósito del desconocimiento de ambas autoridades máximas del Gore de Los Lagos, respecto de las potestades que para formular políticas, y la evidencia que aparece en los diagnósticos sobre este tema hechos por la Subdere, donde el principal instrumento (ERD) para aunar visiones sobre el crecimiento regional, es prácticamente desconocido por las ciudadanía y por los actores relevantes de la sociedad regional. Tomando las palabras de ese diagnostico: "adolece de poder vinculante con la ciudadanía regional y carece de legitimidad".

Por ultimo, se visualiza que las políticas públicas regionales se pueden constituir en instrumentos, que hagan confluir elementos técnicos necesarios para la efectividad de cualquier política, y el debate regional contribuya a que se cimienten acuerdos que otorguen legitimidad al accionar del Estado en los niveles subnacionales. Tal vez la capacidad de generar políticas regionales por parte de los Gores, y su participación en la definición de políticas nacionales que los afecten, sea una mirada que pueda aportar algunos elementos para la discusión sobre la descentralización.

Es posible que el caso estudiado sea único, y no refleje necesariamente la situación de otras regiones relacionadas con otras áreas de actividad, pero dados los antecedentes generales y la forma como está construida la institucionalidad regional y la manera como se relacionan los actores del sistema político chileno, no resulta apresurado aventurar un juicio relativo a que se podría establecer una generalización sobre la escasa participación de los Gores en el diseño y formulación de políticas públicas nacionales con impacto específico y directo en esas regiones.

\section{Referencias bibliográficas}

AGUIRRE, Silvia. Metodología cualitativa en la investigación socio cultural. 1995. Disponible en: <www.lie.upn.mx/docs/ELEMENT008.pdf\#search=\%22Aguirre\% 20Entrevistas\%20y\%20Cuestionarios\%22>. 
ASHER, William. La evolución de las ciencias de las políticas: comprender el surgimiento y evitar la caída. In: VILLANUEVA, Luis Aguilar (Comp.). El estudio de las políticas públicas. México: Editorial Miguel Ángel Porrúa, 1992.

AQUA. Como departamento debemos replantearnos firmemente nuestra estructura operativa. Entrevista realizada a Ricardo Norambuena jefe del Departamento de Acuicultura. Disponible en: <www.aqua.cl/entrevistas/entrevista.php?doc $=19>$. Acceso en 22 ago. 2003.

BID (Banco Interamericano de Desarrollo). Departamento de Investigación. La política de las políticas públicas. v. 8, 2005. Serie Ideas para el Desarrollo de las Américas.

BOISIER, Sergio. La doctrina (oculta) de la descentralización en Chile. Documento preparado para colaborar en el libro Desarrollo regional. Balance de una década de gobiernos regionales. 2003. Disponible en: $<200.80 .149 .114 /$ ecgp/bibliotecavirtual.aspx $>$.

BUSCHMANN, Alejandro. Impacto ambiental de la salmonicultura en Chile: la situación en la X Región de Los Lagos. jun. 2002. Serie Análisis de Políticas Públicas, n. 16.

CAMPOS, Jaime. Discurso inaugural del Seminario Agricultura y Acuicultura. Santiago de Chile, oct. 2003.

CEA EGAÑA, José. El sistema constitucional de Chile. Síntesis crítica. Chile: Facultad de Ciencias Jurídicas y Sociales, Universidad Austral de Chile, 1999.

COLLE, Raymond. La dimensión fáctica del discurso y su evaluación. Revista Latina de Comunicación Social, 2005. Disponible en: <www1.universia.net/CatalogaXXI/ C10010PPESII1/E37963/index.hml>.

COMISIÓN NACIONAL DE ACUICULTURA. Reunión Ordinaria Acta 01. Disponible en: <www.olach.cl/home/olach2/www/images/storiescomision_acuicultura.pdf $>$. Acceso en: 20 ene, 2004.

DE LEON, Peter. Sucesos políticos y las ciencias de políticas. In: WAGNER, Peter; HIRSCHON, Carol; WITTROCK, Bjorn; WOLLMAN, Hellmut (Comp.). Ciencias sociales y Estados modernos. México: Fondo de Cultura Económica, 1999.

DEPARTAMENTO DE ESTUDIOS FUNDACIÓN TERRAM. Pesca y acuicultura: tareas pendientes en la regulación y gestión integral. Serie Análisis de Coyuntura Recursos Naturales, n. 15, mayo 2004.

. Concesiones acuícolas: El agua gratis. Serie Análisis de Coyuntura Salmonicultura, n. 2, ago. 2004.

DOÑA, Karina. Configuración de la agenda de defensa de Chile: análisis del mensaje del gobierno de Chile en el periodo 1990-2005. Chile: Inap/Universidad de Chile 
Publicaciones del Departamento de Gobierno y Gestión Pública. 2006. Disponible en: <www.inap.uchile.cl/gobierno/destacado161.html>.

ECOCEANOS NEWS. Presidente de la República lanza Política Nacional de Acuicultura. Santiago de Chile. Disponible en: <www.ecoceanos.cl/sitio/docs/ cronicas.php? codCronica =18\&codCategoria=1>. Acceso en: 1 ago. 2003.

. La posición de la Subsecretaría de Pesca frente a la Comisión Nacional de Acuicultura. Entrevista a Ricardo Norambuena. Santiago de Chile. Disponible en: <www. ecoceanos.cl/sitio/docs/entrevistas.php?codEntrevista=1> . acceso en: 13 feb. 2004.

ELDER, Charles; COBB, Roger. Formación de la agenda. El caso de la política de los ancianos. In: VILLANUEVA, Luis Aguilar (Comp.). Problemas públicos y agenda de gobierno. México: Editorial Miguel Ángel Porrúa, 1993.

GARZÓN, David. De la ciencia de políticas al análisis de políticas: veinticinco años de progreso. In: VILLANUEVA, Luis Aguilar (Comp.). Problemas públicos y agenda de gobierno. México: Editorial Miguel Ángel Porrúa, 1993.

GOBIERNO DE CHILE. Ministerio del Interior. Diagnostico del proceso de descentralización en Chile. Chile: Subsecretaria de Desarrollo Regional y Administrativo. División de evaluación y cuentas públicas, 2000.

. Ministerio del Interior. El Chile descentralizado que queremos: un proyecto de todos. Chile: Subsecretaria de Desarrollo Regional y Administrativo, 2001.

. Ministerio del Interior. Medición y evaluación del proceso de descentralización. Chile: Subsecretaria de Desarrollo Regional y Administrativo. Informe Final, 2004.

GÓMEZ MENDOZA, Miguel Angel. Análisis de contenido cualitativo y cuantitativo: definición, clasificación y metodología. Revista Ciencias Humanas, n. 20, 2005. Disponible en: <www.utp.edu.co/ chumanas/revistas/revistas/rev20/gomez.htm>.

GOTEES, J. P.; LE COMPTE, M. D. Entonografía y diseño cualitativo en investigación educativa. 1988. Disponible en: <www.ucaldas.edu.co/tsocial/CUADERNILLO5.pdf>.

HECLO, Hugo. Las redes de asuntos y el Poder Ejecutivo. In: VILLANUEVA, Luis Aguilar (Comp.). Problemas públicos y agenda de gobierno. México: Editorial Miguel Ángel Porrúa, 1993.

HIRSCHON, Carol. La investigación de políticas: datos, ideas y argumentos. In: WAGNER, Peter; HIRSCHON, Carol; WITTROCK, Bjorn; WOLLMAN, Hellmut (Comp.). Ciencias sociales y Estados modernos. México: Fondo de Cultura Económica, 1999.

INFANTE, Rodrigo; NEIRA, Roberto. Diagnostico del sector acuícola en Chile. Chile: Ministerio de Economía, Programa de Prospectiva Tecnológica, 2002.

INFORME Económico de la Salmonicultura 2005, Departamento de Estudios SalmonChile, junio, (2006). Disponible en: < http://www.salmonchile.cl/files/InformeEconomico_2005.pdf>. 
LAHERA, Eugenio Parada. Reforma del Estado. In: CONGRESO CHILENO DE ADMINISTRACIÓN PUBLICA, 3., Chile. 4- nov. 1999. 2002 .

. Introducción a las políticas públicas. Chile: Fondo de Cultura Económica,

. Política y políticas sociales. n. 95. Chile: División de Desarrollo Social, Cepal, 2004.

LEY ORGÁNICA CONSTITUCIONAL SOBRE GOBIERNO Y ADMINISTRACIÓN REGIONAL. no 19.175. Disponible en: <www.bcn.cl/publicadores/pub_leyes_mas_ soli/admin/ver_archivo_organica.php?id_organica $=11 \&$ file $=1 \mathrm{c}>$.

LINDBLOM, Charles. El proceso de elaboración de políticas públicas. México: Editorial Miguel Ángel Porrúa, 1991.

LOWI, Theodore. Políticas públicas, estudios de caso y teoría política. In: VILLANUEVA, Luis Aguilar. La hechura de las políticas. México: Editorial Miguel Ángel Porrua, 1992.

MAJONE, Giandomenico. Evidencia, argumentación y persuasión en la formulación de políticas. México: Fondo de Cultura Económica, 1997.

. Programas de investigación y programas de acción o ¿puede la investigación de políticas aprender de la filosofía de la ciencia? In: WAGNER, Peter; HIRSCHON, Carol; WITTROCK, Bjorn; WOLLMAN, Hellmut (Comp.). Ciencias sociales y Estados modernos. México: Fondo de Cultura Económica, 1999.

MEDELLÍN, Pedro. La política de las políticas públicas: propuesta teórica y metodológica para el estudio de las políticas públicas en países de frágil institucionalidad. Chile: Cepal, 2004. (Serie Políticas Sociales).

MELTSNER, Arnold. La factibilidad política y el análisis de las políticas. In: VILLANUEVA, Luis Aguilar. La hechura de las políticas. México: Editorial Miguel Ángel Porrua, 1992.

MINISTERIO DE ECONOMÍA (2002). Prospectiva Chile 2010, la Industria de la Acuicultura. Documento elaborado por el Programa de Prospectiva Tecnológica. Disponible en: <http://www.consejodeinnovacion.cl/cnic/servicios/documentos/ web/download.php?id=74>.

MINISTERIO DEL INTERIOR. Subsecretaria de Desarrollo Regional y Administrativo. Diccionario de administración pública chilena. 2. ed. Santiago, Chile: Editorial LOM Ediciones, 2002.

NEF, Jorge. Política, administración y políticas públicas un análisis sistémico. Revista Política, Santiago, n. 28, 11 jun. 1991.

PAREDES, Olga. Estudio de la industria salmonicultora como conglomerado productivo, a través del modelo de las cinco fuerzas de Porter en la X Región. 2002. Tesis (Magíster 
en Gestión) — Universidad Católica de Valparaíso. Disponible en: <www.comercio. ucv.cl/magister/mpagina/a/doc03control.htm - 39k>.

PÉREZ, Arnaldo Guerra. La industria salmonera. Reportaje en profundidad. Revista Punto Final, 2003. Disponible en: <www.turistel.cl/noticia_ecologia/pag/pag_salmo_neras.htm>.

PIÑUEL, José Luis. Epistemología, metodología y técnicas del análisis de contenido. España: Universidad Complutense de Madrid Departamento de Sociología IV Facultad de CC. de la Información, 2006. Disponible en: <web.jet.es/pinuel. raigada/index.html $>$.

PODESTÁ ARZUBIAGA, Juan. Políticas públicas y regiones. Un análisis crítico. 2003. Disponible en: <www.unap.cl/index.pl?iid $=16744>$.

RIVERA, Claudio Mercado. La discusión de la política de pesca. Chile: Observatorio de Políticas Públicas en Chile, Universidad de Los Lagos, 2003.

SÁNCHEZ, Hugo; REYES, Meza. Metodología y diseños de la investigación científica. Perú: Universidad Ricardo Palma, Editorial Universitaria, 2002.

SUBIRATS, Joan. El análisis de las políticas públicas. España: Departamento de Ciencia Política, Universidad Autónoma de Barcelona, 2001. Disponible en: < www. cnpt.es/docu_pdf/tacticas.pdf>.

SUBSECRETARIA DE PESCA. Anexo legal institucional propuesta Política Nacional Acuícola. Chile. Documento de trabajo versión 2.0, 2002. Disponible en: <www. subpesca.cl>.

. Política Nacional de Acuícola. Documento oficial, 2003a. Disponible en: www.subpesca.cl/mostrararchivo.asp?id=655

. Comunicado aparecido en el portal de esa institución tras la promulgación de la PNA el 3 de agosto el 2003 por el presidente de ese periodo Ricardo Lagos Escobar. 2003b.

TORGERSON, Douglas. Entre el conocimiento y la política: tres caras del análisis de políticas. In: VILLANUEVA, Luis Aguilar (Comp.). El estudio de las políticas públicas. México: Editorial Miguel Ángel Porrúa, 1992.

TUR BONET, José. El pensamiento del profesor ante una situación pluringue. 2005. Tesis (Magíster) — Universidad de Barcelona. Disponible en: <www.sgci.mec.es/redele/ biblioteca2005/tur.shtml>.

VAN METER, Donald; VAN HORN, Carl. El proceso de implementación de las políticas. un marco conceptual. In: VILLANUEVA, Luis Aguilar. La implementación de las políticas. México: Editorial Ángel Porrúa, 1993. 